\title{
A Fixed Point Theorem and an Application to Bellman Operators
}

\author{
Yuhki Hosoya, Masayuki Yao \\ Graduate School of Economics, Keio University, Tokyo, Japan \\ Email: stairlimit@moon.cims.jp,myao@gs.econ.keio.ac.jp
}

Received December 15, 2012; revised January 16, 2013; accepted February 18, 2013

\begin{abstract}
This study introduces a new definition of a metric that corresponds with the topology of uniform convergence on any compact set, and shows both the existence of a unique fixed point of some operator by using this metric and that the iteration of such an operator results in convergence to this fixed point. We demonstrate that this result can be applied to Bellman operators in many situations involving economic dynamics.
\end{abstract}

Keywords: Bellman Operator; Uniform Convergence

\section{Introduction}

Dynamic programming (DP) is an important tool in economic dynamics because many models in which a representative agent maximizes a discounted sum of utilities can be treated as a DP problem. In this context, a fixed point of a Bellman operator plays a significant role and fixed point theorems for contraction mappings (Banach [1]) are usually used for this problem (see Le Van [2], Stokey and Lucas [3]). Recently, fixed point theorems of order-type, such as Knaster-Tarski (e.g., Aliprantis and Border [4], Granas and Dugundji [5]), have also been used for this issue (see Kamihigashi [6], Le Van and Vailakis [7]).

This study treats a fixed point theorem of the former. However, the metric we use is different from those in past research. Although most related research uses the uniform norm as the metric, we treat a new metric that corresponds with the topology of uniform convergence on any compact set. Our main results focus on two points. First, we show that there exists a unique fixed point of some operator. Second, we show that the iteration of such an operator results in convergence to this fixed point. This fixed point theorem can be applied Bellman operators in many dynamic economic systems.

The rest of the paper is organized as follows. In the next section, we introduce our framework and state our basic result. In Section 3, we present an application of our theorem to Bellman operators. In the appendix, we give an additional result on our metric.

\section{Framework and Basic Results}

Let $X$ be a Hausdorff space and suppose that there exists an increasing sequence $\left(K_{n}\right)$ of compact sets in $X$ such that $^{1}$

$$
X=\bigcup_{n} \operatorname{int} K_{n}
$$

For any real-valued functions $f, g$ on $X$, let

$$
d_{n}(f, g)=\sup _{x \in K_{n}}|f(x)-g(x)|
$$

and let $F(X)$ be the set of all functions such that $d_{n}(f, 0)<+\infty$. Then $d_{n}$ is a pseudo-metric on $F(X)$. Define

$$
d(f, g)=\sum_{n} \frac{1}{2^{n}} \arctan \left(d_{n}(f, g)\right)
$$

for any $f, g \in F(X)$ and $d$ is a metric of $F(X)$. In the Appendix, we will verify that, for any sequences $\left(f_{m}\right) \in F(X)$ and $f \in F(X), d\left(f_{m}, f\right) \rightarrow 0$ if and only if $f_{m}$ converges to $f$ uniformly on any compact subset of $X$.

The following theorem holds.

Theorem 1: Suppose that $T: F(X) \rightarrow F(X)$ satisfies the following two conditions:

1) For any $n$ and any $x \in K_{n}, T f(x) \leq T g(x)$ if $f(y) \leq g(y)$ for any $y \in K_{n}$;

2) There exists $\beta \in] 0,1[$ such that, for any $x \in X$ and $a \geq 0, T(f+a)(x) \leq T f(x)+\beta a$.

Choose any $f \in F(X)$ and define $f_{0}=f$ and $f_{m}=T f_{m-1}$ for any $m \geq 1$. Then $f_{m}$ converges to a unique fixed point $f^{*}$ of $T$ with respect to $d$.

Proof: Choose any $g, h \in F(X)$. By definition of $d_{n}(g, h)$, we have

${ }^{1}$ Such a sequence exists if $X$ is locally compact and second countable. 


$$
g(x) \leq h(x)+d_{n}(g, h)
$$

for any $x \in K_{n}$. By (1) and (2),

$$
T g(x) \leq T\left(h+d_{n}(g, h)\right)(x) \leq T h(x)+\beta d_{n}(g, h)
$$

Hence, we have $T g(x)-T h(x) \leq \beta d_{n}(g, h)$. By symmetry, we can verify that $T h(x)-T g(x) \leq \beta d_{n}(g, h)$. Thus,

$$
|\operatorname{Tg}(x)-\operatorname{Th}(x)| \leq \beta d_{n}(g, h)
$$

for all $x \in K_{n}$, and hence

$$
d_{n}(T g, T h) \leq \beta d_{n}(g, h)
$$

for any $\$ \mathrm{n} \$$. Therefore, if $g \neq h$,

$$
\begin{aligned}
d(T f, T g) & =\sum_{n} \frac{1}{2^{n}} \arctan \left(d_{n}(T f, T g)\right) \\
& \leq \sum_{n} \frac{1}{2^{n}} \arctan \left(\beta d_{n}(f, g)\right) \\
& <\sum_{n} \frac{1}{2^{n}} \arctan \left(d_{n}(f, g)\right) \\
& =d(g, h)
\end{aligned}
$$

If $g, h \in F(X)$ are two distinct fixed points of $T$, then

$$
d(g, h)=d(T g, T h)<d(g, h),
$$

which is a contradiction. Thus, $T$ has at most one fixed point $^{2}$.

Next, for any $n \geq N$ and $m, k \geq 0$,

$$
\begin{aligned}
d_{n}\left(f_{m}, f_{m+k}\right) & =\sum_{i=1}^{k} d_{n}\left(f_{m+i}, f_{m+i-1}\right) \\
& \leq d_{n}\left(f_{m}, f_{m+1}\right) \sum_{i=1}^{k} \beta^{i-1} \\
& \leq \frac{\beta^{m}}{1-\beta} d_{n}(f, T f) .
\end{aligned}
$$

Therefore, if $x \in K_{n}$, then

$$
\left|f_{m}(x)-f_{m+k}(x)\right| \leq \frac{\beta^{m}}{1-\beta} d_{n}(f, T f)
$$

and thus $\left(f_{m}(x)\right)$ is a Cauchy sequence. Hence, $f_{m}(x)$ converges to some real number denoted by $f^{*}(x)$. Then

\footnotetext{
${ }^{2}$ Let $(M, \rho)$ be a metric space. A mapping $T: M \rightarrow M$ is said to be contractive (strictly contractive) if $\rho(T(x), T(y))<\rho(x, y)$ for each $x, y \in M$ with $x \neq y$. In our result, we use this mapping, but the following well-known result (for a detailed argument, see Goebel and Kirk [8], Kirk [9]) is not applicable since it assume a compact metric space.

Theorem: Let $(M, \rho)$ be a compact metric space and let $T: M \rightarrow M$ be a contractive mapping. Then $T$ has a unique fixed point $v$, and moreover, for each $x \in M, \lim _{m \rightarrow \infty} T^{m} x=v$.
}

$$
\left|f(x)-f^{*}(x)\right| \leq \frac{1}{1-\beta} d_{n}(f, T f),
$$

and thus

$$
\begin{aligned}
d_{n}\left(f^{*}, 0\right) & \leq d_{n}\left(f^{*}, f\right)+d_{n}(f, 0) \\
& \leq \frac{1}{1-\beta} d_{n}(f, T f)+d_{n}(f, 0) \rightarrow+\infty
\end{aligned}
$$

Hence, the function $f^{*}$ is in $F(X)$.

Now, for any $n$,

$$
0 \leq d_{n}\left(f_{m}, f^{*}\right) \leq \frac{\beta^{m}}{1-\beta} d_{n}(f, T f) \rightarrow 0
$$

as $m \rightarrow \infty$, and thus $d_{n}\left(f_{m}, f^{*}\right) \rightarrow 0$ for any $n$. Choose any $\varepsilon>0$, and choose any $k$ such that $\pi / 2^{k} \leq \varepsilon$. We have already shown that, for any sufficiently large $m$,

$$
d_{n}\left(f_{m}, f^{*}\right)<\frac{\varepsilon}{2}
$$

for all $n \in\{1, \cdots, k\}$. Then ${ }^{3}$

$$
\begin{aligned}
d\left(f_{m}, f^{*}\right) & =\sum_{n} \frac{1}{2^{n}} \arctan \left(d_{n}\left(f_{m}, f^{*}\right)\right) \\
& <\sum_{n=1}^{k} \frac{\varepsilon}{2^{n+1}}+\sum_{n} \frac{1}{2^{n}} \arctan \left(d_{n}\left(f_{m}, f^{*}\right)\right) \\
& <\frac{\varepsilon}{2}+\frac{\pi}{2^{k}} \sum_{n=1}^{\infty} \frac{1}{2^{n+1}} \leq \varepsilon
\end{aligned}
$$

and thus $d\left(f_{m}, f^{*}\right) \rightarrow 0$.

Now, $T$ is a Lipschitz function on $d$ and is thus continuous. Hence, $T f_{m} \rightarrow T f^{*}$. Meanwhile, since $T f_{m}=f_{m+1}, T f_{m} \rightarrow T f^{*}$. Thus $T f^{*}=f^{*}$, and so $f^{*}$ is a fixed point of $T$. This completes the proof.

\section{Application to Bellman Operators}

Let $X$ be a Hausdorff space, let $\Gamma$ be a correspondence from $X$ into $X$ and let $u$ be a real-valued function on $\left\{(x, y) \in X^{2} \mid y \in \Gamma(x)\right\}$, and define

$$
B f(x)=\sup \{u(x, y)+\beta f(y) \mid y \in \Gamma(x)\} .
$$

We call the operator B a Bellman operator. Consider the following problem:

$$
\begin{array}{cc}
\max & \sum_{t=0}^{\infty} \beta^{t} u\left(x_{t}, x_{t+1}\right) \\
\text { subject to } & x_{t+1} \in \Gamma\left(x_{t}\right) \\
& x_{0} \text { is given. }
\end{array}
$$

Let $V\left(x_{0}\right)$ denote the maximum value for the above problem. It is well-known that under several conditions, $V$ is a fixed point of the Bellman operator.

${ }^{3}$ Note that $\arctan (x) \leq x$ for any $x \geq 0$. 
Then we can show the following theorem.

Theorem 2: Suppose that

1) $\bar{u}(x) \equiv \sup _{y \in \Gamma(x)} u(x, y)$ is real-valued and continuous on $X$;

2) $\Gamma(x) \subset K_{n}$ for any $x \in K_{n}$.

Then $B$ is a mapping from $F(X)$ into $F(X)$. Further, for any $f \in F(X)$, if $f_{0}=f$ and $f_{m}=B f_{m-1}$ for any $m \geq 1$, then $f_{m}$ converges to a unique fixed point of $B$ with respect to $d$.

Note that the conditions of Theorem 2 are not so strict. In many economic models, the following conditions are satisfied:

1) $X=\mathbb{R}_{+}$;

2) There exists $\bar{x} \in X$ such that, if $x \geq \bar{x}$, then $y \leq x$ for any $y \in \Gamma(x) \$$;

3) For any $x \in X, 0 \in \Gamma(x)$;

4) $u(x, y)$ is non-increasing in $y$;

5) $u(x, 0)$ is continuous in $x$.

Under these conditions, we can show that $\bar{u}(x)=u(x, y)$, and thus condition 1) of Theorem 2 is satisfied. Also, by setting $K_{n}=\{x \in X \mid 0 \leq x \leq \bar{x} n\}$, condition 2) of Theorem 2 is satisfied. Hence Theorem 2 is applicable.

Proof: By 2), B satisfies 1) and 2) of Theorem 1. Hence, it suffices to show that $B$ is a mapping from $F(X)$ into $F(X)$. By 1 ), we have $B 0=\bar{u} \in F(X)$. Choose any $f \in F(X)$. As in the proof of Theorem 1 , we can show that

$$
d_{n}(B f, B 0) \leq \beta d_{n}(f, 0) .
$$

Then

$$
\begin{aligned}
d_{n}(B f, 0) & \leq d_{n}(B f, B 0)+d_{n}(B 0,0) \\
& \leq \beta d_{n}(f, 0)+d_{n}(B 0,0)<+\infty
\end{aligned}
$$

which implies that $B f \in F(X)$. This completes the proof.

\section{Conclusion}

In this paper, we introduced a new fixed point theorem and showed that it can be applied to the Bellman operator of several economic models. The claim of our theorem includes not only the existence of fixed point but also the convergence result on iteration. By using our result, one can get value function from iterative application of the Bellman operator in a wide class of dynamic economic models.

\section{Acknowledgements}

The authors are grateful to Hiroyuki Ozaki for his helpful comments and suggestion. This research is partially supported by Keio/Kyoto Joint Global Center of Excellence Program Raising Market Quality-Integrated Design of "Market Infrastructure".

\section{REFERENCES}

[1] S. Banach, "Sur les Operations dans les Ensembles Abstraits et Leur Applications aux Equations Integrals," Fundamenta Mathematicae, Vol. 3, 1922, pp. 133-181.

[2] C. Le Van, "Optimal Growth Models with Discounted Return," In: R.-A. Dana, C. Le Van and K. Nishimura, Eds., Handbook on Optimal Growth 1 Discrete Time, Springer Berlin, Heidelberg, 2006, pp. 19-54. doi:10.1007/3-540-32310-4_2

[3] N. Stokey and R. E. Lucas Jr. and E. C. Prescott, "Recursive Methods in Economic Dynamics," Harvard University Press, Cambridge, 1989.

[4] C. D. Aliprantis and K. C. Border, "Infinite Dimensional Analysis: A Hitchhiker's Guide," 3rd Edition, SpringerVerlag, Berlin, 2006.

[5] A. Granas and J. Dugundji, "Fixed Point Theory," Springer-Verlag, New York, 2003. doi:10.1007/978-0-387-21593-8

[6] T. Kamihigashi, "Elementary Results on Solutions to the Bellman Equation of Dynamic Programming: Existence, Uniqueness, and Convergence," Discussion Paper Series, No. DP2012-31, RIEB Kobe University, Kobe, 2012.

[7] C. Le Van and Y. Vailakis, "Monotone Concave (Convex) Operators: Applications to Stochastic Dynamic Programming with Unbounded Returns," Memeo, University of Paris 1 and Iniversity of Exeter Business School, Paris, 2011.

[8] K. Goebel and W. A. Kirk, "Topics in Metric Fixed Point Theory," Cambridge University Press, Cambridge, 1990. doi:10.1017/CBO9780511526152

[9] W. A. Kirk, "Contraction Mappings and Extensions," In: W. A. Kirk and B. Sims, Eds., Handbook of Metric Fixed Point Theory, Kluwer Academic Publishers, Dordrecht, 2001, pp. 1-34. 


\section{Appendix. Additional Notes on Our Metric}

In this section, we prove the following theorem.

Theorem A: Suppose $X$ satisfies the assumption in Section 2 and we define $d^{N}$ as in Section 2. Suppose also that $\left(f_{m}\right)$ is a sequence in $F(X)$ and that $f \in F(X)$. Then $d\left(f_{m}, f\right) \rightarrow 0$ if and only if

$$
\sup _{x \in K}\left|f_{m}(x)-f(x)\right| \rightarrow 0
$$

for any compact set $K \subset X$.

Proof of Theorem A: If the latter holds, then we have $d_{n}\left(f_{m}, f\right) \rightarrow 0$ for any $n$. Therefore,

$$
d^{N}\left(f_{m}, f\right) \rightarrow 0 .
$$

Conversely, suppose that $d^{N}\left(f_{m}, f\right) \rightarrow 0$. For any compact set $K \subset X$, (int. $\left.K_{n}\right)$ is an open covering of $K$, and thus there exist $n_{1}, \cdots, n_{k}$ such that

$$
K \subset \bigcup_{i=1}^{k} K_{n_{i}}
$$

Since $d\left(f_{m}, f\right) \rightarrow 0$, we have $d_{n_{i}}\left(f_{m}, f\right) \rightarrow 0$. Therefore,

$$
0 \leq \sup _{x \in K}\left|f_{m}(x)-f(x)\right| \leq \max _{i=1, \cdots, k} d_{n_{i}}\left(f_{m}, f\right) \rightarrow 0,
$$

which completes the proof. $\square$ 